ISSN: 2386-3919 - e-ISSN: 2386-3927

DOI: https://doi.org/10.14201/et2018362729

\title{
USO EDUCATIVO DE LA RA: EXPERIENCIAS EN ESPAÑA Y MÉXICO
}

\section{Educational use of RA: experiences in Spain and Mexico}

Julio Barroso-Osuna*, Julio CABERO-AlmenarA** y Rubicelia VAlENCIA ORTIZ****

*Universidad de Sevilla-España

Correo-e:jbarroso@us.es

* Universidad de Sevilla-España

Correo-e: cabero@us.es

*** Instituto Latinoamericano de Comunicación Educativa-México

Correo-e: Rubi.valencia@ilce.edu.mx

Recibido: 01/09/2018; Aceptado: 07/11/2018; Publicado: 30/12/2018

Ref. Bibl. JULIO BARROSO-OSUNA, JULIO CABERO-ALMENARA y RUBICELIA VALENCIA ORTIZ. Uso educativo de la RA: experiencias en España y México. Enseñanza \& Teaching, 36, 2-2018, 7-29.

RESUMEN: Desde los últimos estudios publicados, se apunta hacia la Realidad Aumentada (RA), como una tecnología de fuerte impacto en la educación, en diferentes áreas de conocimiento y niveles educativos. En este artículo se describen dos experiencias de su incorporación en niveles educativos diferentes, una en niveles universitarios y otra en el nivel de infantil y primaria. La primera de ellas se refiere a una investigación multicaso, mientras que la siguiente se trata de una propuesta de innovación en los niveles anteriormente citados. Los resultados obtenidos en los dos estudios presentados nos permiten corroborar los alcanzados en otras investigaciones realizadas, encontrándonos ante una tecnología que puede ser utilizada en diferentes áreas y niveles educativos, si bien para la mayoría de los casos se hace necesaria la creación de centros de producción de estos objetos, sin perder de vista que para su verdadera integración curricular es fundamental un buen diseño pedagógico y la realizaciones de investigaciones que nos aporten pistas sobre su diseño y utilización en el campo de la educación. 
Palabras clave: Realidad Aumentada; innovación educativa; formación universitaria; tecnologías emergentes.

SUMMARY: Since the last published studies, it points to Augmented Reality (AR), as technology that will strongly impact education, in different educational levels and areas. This article describes two experiences of its incorporation into a variety of educational levels, one at the university level and the other at the infant and primary level. The first one refers to a multicase research, meanwhile the next one presents a proposal for innovation in the aforementioned levels.

The results obtained in the two studies presented, allow us to corroborate those achieved in other investigations, finding ourselves faced with technology that can be used in different educational areas and levels, although for the majority of the cases it is imperative to create production centers for these objects, without losing sight of their true curricular integration, sound pedagogical design it as is carried out some research that provide us with clues about their design and use in the field of education.

Key words: Augmented Reality; educational innovation; university education; emerging technologies.

\section{INTRODUCCIÓN}

Dentro de las tecnologías que están penetrando últimamente en la sociedad en general, y en la educación y formación, en particular, nos encontramos con la «Realidad Aumentada" ("Augmented Reality») (RA). Y ello es debido, entre otros motivos, a que los dispositivos usuales para su visionado, tablets y smartphones, encuentran una fuerte presencia en los usuarios, lo que repercute en favorecer el aprendizaje móvil y ubicuo (Sevillano y Vázquez-Cano, 2015; Vázquez-Cano y Sevillano, 2015).

En el presente artículo se describen dos experiencias de su incorporación centradas en niveles educativos diferentes y con propuestas de acción distintas; una se mueve en la enseñanza universitaria y es específicamente una investigación multicaso, y otra es una propuesta de innovación y se moviliza en el nivel de infantil y primaria.

\section{LA ReAlidad Aumentada: TeCNOlOgía EMERGENTE PARA LA FORMACiÓN}

Como hemos señalado, la RA se presenta como una tecnología emergente con fuerte penetración en el contexto formativo en un horizonte no muy lejano, como claramente ponen de manifiesto los Proyectos Horizon (Adams y otros, 2016; Johnson y otros, 2016) o los Informes EduTrend del Tecnológico de Monterrey (Tecnológico de Monterrey, 2015 y 2017).

Ahora bien, ¿qué podemos entender por RA? Y al respecto digamos que se trata de una tecnología que pretende combinar información digital e información física 
en tiempo real a través de diferentes dispositivos tecnológicos; es decir, consiste en utilizar un conjunto de dispositivos tecnológicos que añaden información virtual a la información física, por tanto, implica añadir una parte sintética virtual a lo real (Cabero y García, 2016).

Para De la Torre Cantero y otros $(2013,5)$ : «es una tecnología que permite la interacción del usuario con el mundo físico y real que lo rodea. La RA combina tres dimensiones (3D) de objetos generados por ordenador y texto superpuesto sobre imágenes reales y vídeo, todo en tiempo real». Y que, como señalan Di Serio y otros $(2013,587)$, es una tecnología que presenta tres características definitorias: combinar objetos reales y virtuales en un entorno real; alineación de objetos reales y virtuales entre sí, y ejecutarlos de forma interactiva y en tiempo real. Diferenciándose de la realidad mixta y virtual en que los alrededores y los objetos virtuales son visibles (Brigham, 2017).

En definitiva, lo que persigue es enriquecer la información existente en la realidad con información disponible en los dispositivos tecnológicos; de manera que se cree una nueva tecnología que permite completar y aumentar la información real con información digital.

Para su producción son necesarias diferentes tecnologías y recursos, que podemos concretar en los siguientes:

1. Un elemento que capture la imagen de la realidad que están viendo los usuarios (pantalla del ordenador, un teléfono o una videoconsola);

2. Un dispositivo donde proyectar la mezcla de las imágenes reales con las imágenes sintetizadas (pueden servir los tres citados anteriormente);

3. Un elemento de procesamiento o varios que trabajen conjuntamente cuya función es la de interpretar la información del mundo real que recibe el usuario, generar la información virtual que cada servicio concreto necesite y mezclarla de forma adecuada (ordenadores, móviles o videoconsolas);

4. Un tipo de software específico para la producción del programa;

5. Un activador de la realidad aumentada o marcadores que pueden ser códigos QL, objetos físicos, GPS ...); y

6. Un servidor de contenidos donde se ubica la información virtual que queremos incorporar a la realidad (Cabero y García, 2016; Cabero y otros, 2016; Villalustre y Del Moral, 2016; Cabero, De la Horra y Sánchez, 2018).

Su penetración rápida en el sistema educativo se debe a que ofrece una serie de posibilidades, que podemos concretar de acuerdo con las propuestas de diferentes autores. Su utilización en la formación, como señalan diferentes autores (Wu, Wen-Yu, Chang y Liang, 2013; Carozza, Tingdahl y Gool, 2014; Han, Jo, Hyun y So, 2015; Prendes, 2015; Santos, Wolde, Taketomi, Yamamoto, Rodrigo, Sandor y Kato, 2016; Villalustre y Del Moral, 2016; Aguayo, Cochrane y Narayan, 2017; Pejoska-Laajola, Reponen, Virnes y Leinonen, 2017; Chang y Hwang, 2018; Ibáñez y Delgado, 2018; Piqueras y González-Calero, 2018), permite diferentes posibilidades como son: a) eliminar información que pueda entorpecer la captación de la 
información significativa por el estudiante; b) aumentar o enriquecer la información de la realidad para hacerla más comprensible al estudiante; c) poder observar un objeto desde diferentes puntos de vista seleccionando el estudiante el momento y posición de observación; d) potencia el aprendizaje ubicuo; e) crear escenarios "artificiales» seguros para los estudiantes como pueden ser laboratorios o simuladores; f) enriquecer los materiales impresos para los estudiantes con información adicional en diferentes soportes; g) convertir a los alumnos en "proconsumidores" de objetos de aprendizaje en formato RA; h) puede ser utilizada en acciones de formación apoyadas en la metodología del flipped classroom; i) se potencia el aprendizaje informal, y j) que el estudiante permanece en el mundo real y, por tanto, no pierde la contextualización, lo que propicia la contextualización de la información. A ellas debemos incorporarles que puede ser utilizada en diferentes disciplinas y en distintos niveles educativos (Bressler y Bodzin, 2013; Akçayır y Akçayır, 2017), aunque es en la formación universitaria donde se está utilizando con mayor frecuencia (Lin, Been-Lirn, Li, Wang y Tsai, 2013).

Por otra parte, debemos señalar que diferentes estudios e investigaciones han puesto de manifiesto que los alumnos pueden convertirse en productores de objetos de aprendizaje en RA, lo que propicia la adquisición no solo de los productos por parte de los estudiantes, sino, lo que es más significativo, los procesos que los rigen. (Barroso y Gallego, 2017; Gallego y otros, 2018; Martínez y Fernández, 2018).

Frente a las posibilidades que ofrece, posee también una serie de limitaciones que presenta esta tecnología para ser incorporada a la formación, las cuales, sin el ánimo de acotar el tema, las podemos concretar en las siguientes:

- Hay más propuestas tecnológicas que prácticas educativas de su incorporación.

- La novedad está llevando a una falta de reflexión teórica. Falta de modelos teóricos con los cuales podemos analizar, por una parte, su incorporación a la enseñanza y, por otra, la búsqueda de estrategias de utilización.

- Faltan materiales educativos adecuados al currículum.

- Formación del profesorado.

- Y el poco volumen de investigaciones que se han desarrollado.

Indicar que en el presente artículo pretendemos ofrecer algunas aportaciones para paliar algunas de las limitaciones que se han apuntado. Pero señalaremos dos aspectos.

En primer lugar, que es una tecnología que se está incorporando a los diferentes niveles de enseñanza, que van desde primaria (Bongiovani, 2013; Villalustre y Del Moral, 2016; Cabero y otros, 2018) a secundaria-bachillerato-formación profesional (Avendaño y otros, 2013; De la Torre y otros, 2013; Di Serio y otros, 2013; Kamarainen y otros, 2013; Cabero y otros, 2018). Aunque es en la enseñanza universitaria donde se han llevado a cabo más experiencias e investigaciones (Cabero, Fernández y Marín, 2017; Marín, 2017; Fernández, 2018). 
Y en segundo lugar, que es una tecnología que se está incorporando a distintas áreas de conocimiento y disciplinas, que incluyen la ingeniería, las matemáticas, las ciencias de la educación, geografía o la medicina (Chen y Wang, 2015; Barroso y Cabero, 2016; Barroso y otros, 2016; Akcayr y Akcayr, 2017; Martínez y Fernández, 2018). Aplicaciones que pueden observarse con más detalle en los recientes metanálisis publicado por Tekederea y Gökera (2016), referido a las aplicaciones de la RA en el terreno educativo.

\section{APORTACIONES DESDE EL PROYECTO DE INVESTIGACIÓN RAFODIUN}

Este proyecto de investigación fue concebido con el objeto de analizar las posibilidades que la RA tiene para la formación universitaria. Presentamos un proyecto de investigación a la convocatoria de I+D del Ministerio de Economía y Competitividad del Gobierno de España denominado: "Realidad aumentada para aumentar la formación. Diseño, producción y evaluación de programas de realidad aumentada para la formación universitaria» (EDU-5746-P-Proyecto Rafodiun), que fue aprobado y que persigue los siguientes grandes objetivos:

1. Evaluar las posibilidades y potencialidades que ofrecen diferentes softwares utilizados para la creación de entornos tecnológicos bajo la arquitectura de la RA para ser utilizados en contextos formativos universitarios.

2. Analizar las posibilidades que los diferentes tipos de dispositivos de RA ofrecen para su aplicación en contextos de enseñanza universitaria.

3. Diseñar y producir distintos contenidos en formato RA para ser aplicados en contextos de formación universitaria en distintas áreas curriculares, y evaluar sus posibilidades de cara al rendimiento.

4. Conocer el grado de motivación y nivel de satisfacción que despierta en los estudiantes universitarios el hecho de participar en experiencias formativas apoyadas en RA.

5. Crear un entorno formativo bajo la arquitectura de la RA, en formato libro electrónico, para la capacitación del profesorado universitario en el diseño, producción y utilización educativa de la RA.

6. Poner en acción y validar el entorno producido para la capacitación del profesorado universitario en el diseño, producción y utilización educativa de la RA.

7. Conocer las posibilidades educativas que permite que el alumno se convierta en productor de experiencias formativas apoyadas en la RA.

8. Indagar sobre las dificultades técnicas, curriculares y organizativas que pudiera tener la RA para ser aplicada a los contextos de formación universitaria.

9. Y crear una comunidad virtual formada por profesorado universitario preocupado por la utilización educativa de la RA. 
Con ellos lo que se pretendía es analizar las posibilidades educativas que para contextos de formación universitaria puede tener la RA. Y este análisis lo efectuaremos desde diferentes perspectivas tanto tecnológico-instrumentales, como educativas, de diseño de entornos formativos, hasta la referida a la formación y el perfeccionamiento del profesorado, y al alumno como productor de contenidos en RA. El proyecto comenzó en junio del 2015 y finalizó en diciembre de 2017.

El alcance de los diferentes objetivos se llevó a cabo mediante diferentes tipos de técnicas y estrategias usualmente utilizadas en la investigación en el terreno de la Tecnología Educativa (Barroso y Cabero, 2010), y a través de diferentes fases:

Primera Fase: Evaluación y análisis del software de producción de la RA y de los diferentes tipos de dispositivos.

En esta primera fase se realizaron diferentes actividades encaminadas a evaluar los diferentes programas de software informáticos existentes en el mercado para la producción de entornos de RA, así como la evaluación de los diversos tipos de dispositivos que se pueden utilizar para la producción de entornos en RA. Con ello pretendimos dar respuesta a los objetivos 1 y 2 señalados anteriormente.

Etapa 1. Evaluación del software informático de producción de entornos RA. En esta fase se realizaron las siguientes actividades:

a) Revisión e identificación de los principales programas informáticos que existen en el mercado para la producción de contenidos en RA.

b) Configuración de una lista de programas con sus direcciones web e identificación de versiones de prueba de los programas de producción de RA.

c) Creación de una lista de chequeo para la evaluación de los programas identificados.

d) Elaboración de la versión en Internet del instrumento.

e) Evaluación del software mediante la aplicación de la técnica del juicio de experto.

Etapa 2. Análisis de las posibilidades que los diferentes tipos de dispositivos de RA ofrecen para su aplicación en contextos de enseñanza universitaria.

Y para ello se realizaron las siguientes actividades.

a) Configuración de grupos de discusión.

b) Realización de los grupos de discusión, que se realizaron de acuerdo a la siguiente estructura: introducción por el moderador de los objetivos que se persiguen con la actividad y de los objetivos que perseguimos con nuestro trabajo; presentación de las personas que conforman el grupo de discusión; exposición de los diferentes dispositivos; aclaración de dudas sobre los objetivos y los dispositivos utilizados en la RA, y realización de la sesión. 
Etapa 3. Creación de una comunidad virtual de profesorado universitario preocupado por la utilización educativa de la RA.

FIGURA 1

Comunidad virtual RAFODIUN

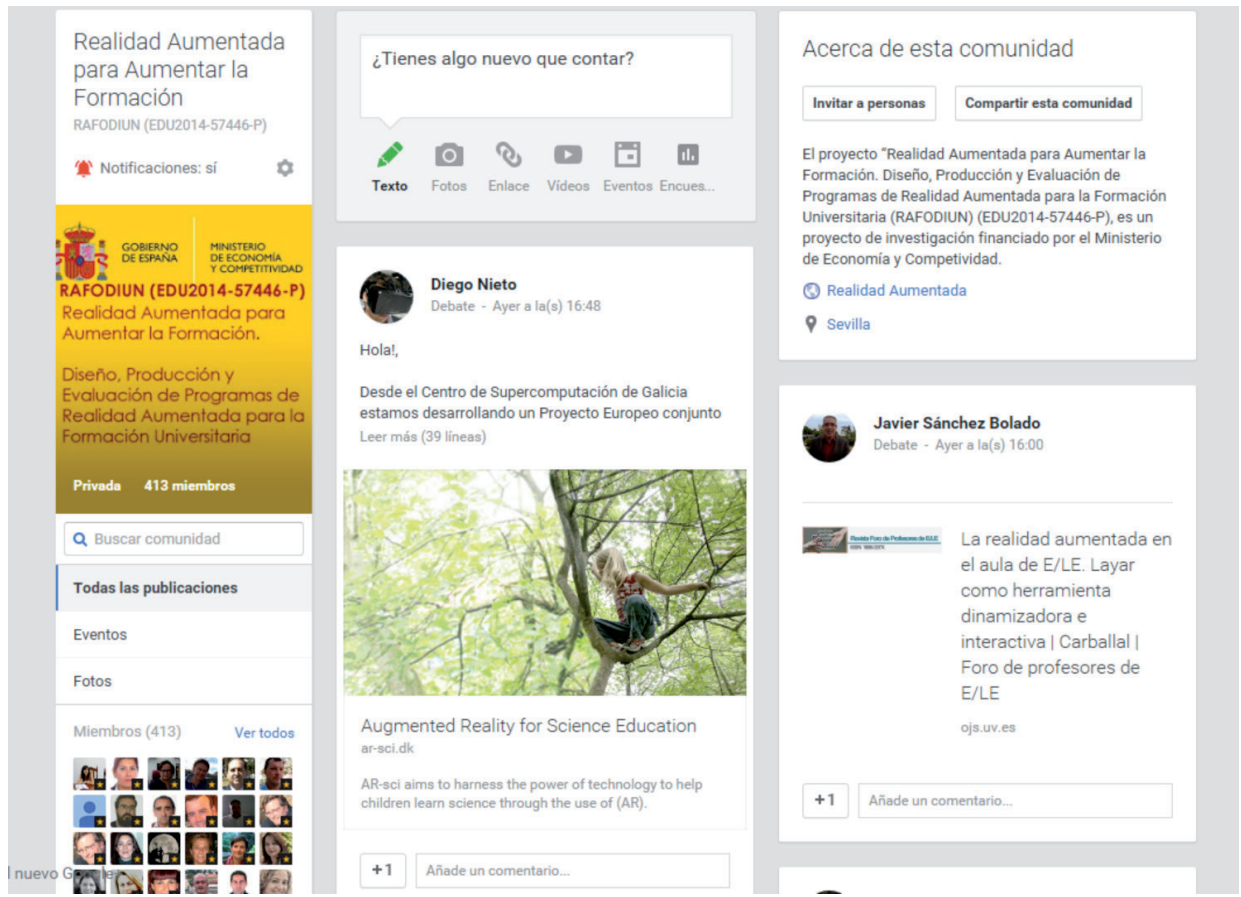

Segunda Fase: Diseño y producción de contenidos formativos en formato RA que se aplicaron en contextos de formación universitaria.

Para la producción de los contenidos en diferentes formatos de RA, se siguió el siguiente procedimiento: selección de los contenidos, producción de los contenidos en RA, producción técnica a través de diferentes herramientas y evaluación mediante un grupo de expertos y diferentes estudios piloto.

Con los diferentes estudios piloto, pretendíamos conocer si los alumnos, tras interaccionar con los contenidos elaborados en RA, aprendían los conceptos presentados en los mismos. Entendiendo en este caso por aprender la capacidad de recordar, comprender y aplicar la información presentada. Para ello y teniendo en cuenta la taxonomía de Bloom para la era digital (Churches, 2008), se administró un cuestionario a los estudiantes participantes en el estudio que cursaban la asignatura de tecnología educativa, en el Grado de Pedagogía y Maestro de Primaria en la 
Universidad de Sevilla, del tipo de elección múltiple, administrado en la modalidad de pretes y postest.

Para alcanzar este objetivo se construyeron diferentes tipos de objetos de aprendizaje para distintas disciplinas y áreas de conocimiento; que fueron la medicina, la pedagogía, la expresión musical, las bellas artes y la arquitectura, y que pertenecían a contenidos curriculares que se impartían en asignaturas de estas disciplinas. Estos objetos pueden observarse en la siguiente dirección: http://grupotecnologiaeducativa.es/proyectorafodiun/index.php/objetos-en-ra. En la figura 2 se presentan algunos de ellos.

FIGURA 2

Imágenes de diferentes objetos producidos en RA
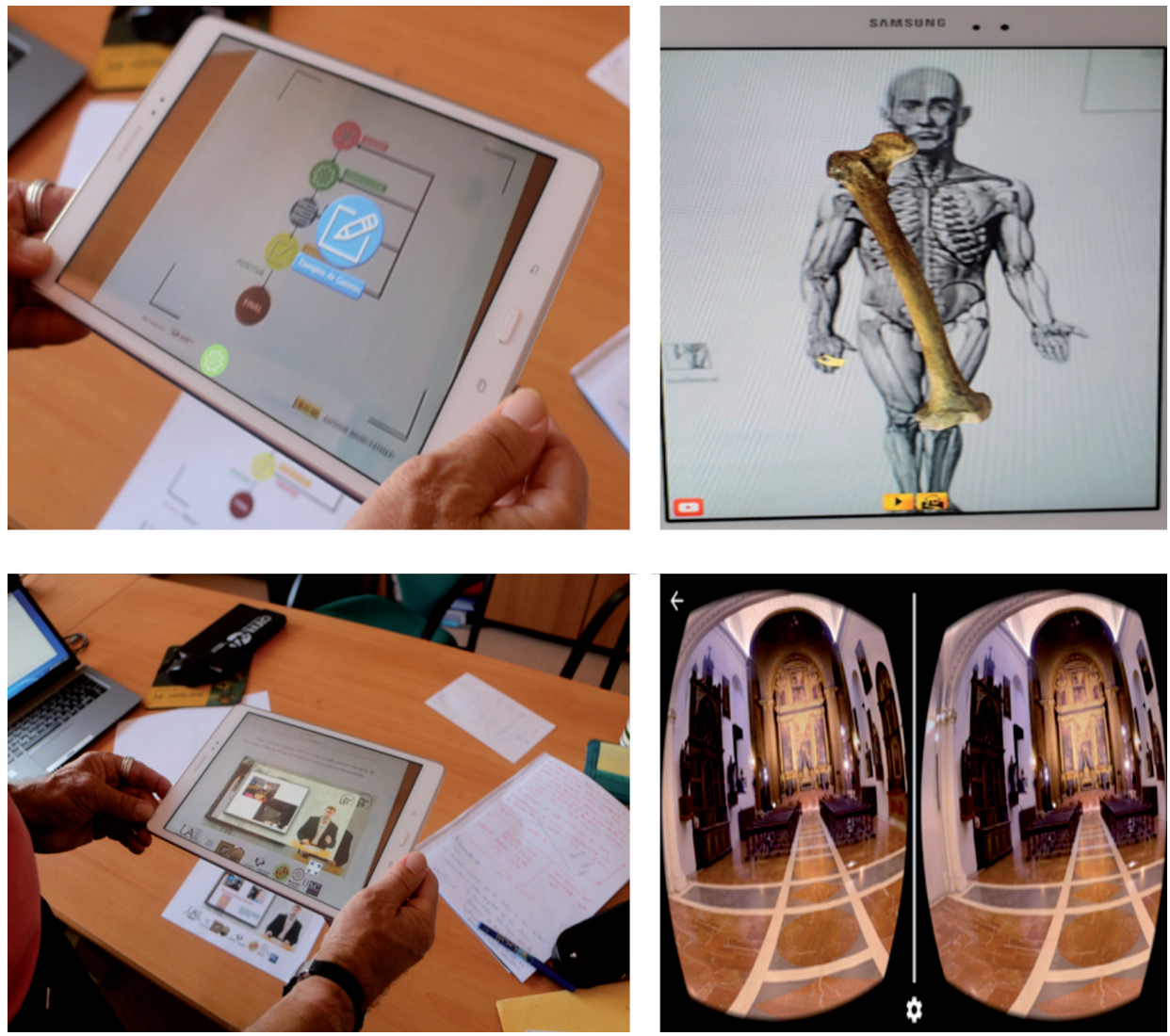

Para la producción de estos objetos se utilizaron diferentes programas informáticos (Tabla 1). 
TABLA 1

Programas utilizados para la producción de los objetos

\begin{tabular}{|c|c|}
\hline SOFTWARE & PARA QUÉ SE UTILIZA \\
\hline Metaio Creator & Programación realidad aumentada. \\
\hline Metaio SDK & Kit de desarrollo de software RA. \\
\hline Eclipse & $\begin{array}{c}\text { Entorno de desarrollo Java. Exportación APK para android. } \\
\text { IPA para ios. Subida a la app store. }\end{array}$ \\
\hline Xcode & $\begin{array}{c}\text { Postproducción de vídeo y sonido. } \\
\text { Chroma, Rotobrush, Key Light. }\end{array}$ \\
\hline Adobe After effects & $\begin{array}{c}\text { Postproducción de imagen. Grafismos. } \\
\text { Photomerge. Texturizado 3D. }\end{array}$ \\
\hline Adobe Photoshop & Postproducción de imagen. Grafismos. \\
\hline Macromedia Fireworks & Augment----RA. \\
\hline Ffmpeg & Programación sobre el codec para exportación vídeos 3g2. \\
\hline MS Powerpoint & Editor profesional de texto para retoque de código. \\
\hline Notepad ++ & Extracción marcadora basado en 3D. \\
\hline Metaio toolbox & Fotogrametría. \\
\hline Autocatch123D & Escaneo 3D. \\
\hline Artec Studio & Creador de instalador windows. \\
\hline Astrum &
\end{tabular}

Con dichos objetos se llevaron a cabo diferentes estudios, midiendo las diferentes variables con los instrumentos que se presentan en la Tabla 2. 
TABLA 2

Instrumentos de recogida de información

\begin{tabular}{|c|c|}
\hline VARIABLE & INSTRUMENTO DE EVALUACIÓN \\
\hline Rendimiento académico & $\begin{array}{c}\text { Instrumento «ad hoc", con construcción de } \\
\text { prueba de elección múltiple, y utilizado } \\
\text { bajo la modalidad pretest-postest. }\end{array}$ \\
\hline Nivel de aceptación de la tecnología & $\begin{array}{c}\text { "Technology Acceptance Model» } \\
\text { (TAM) (Davis, 1989). }\end{array}$ \\
\hline Motivación & $\begin{array}{c}\text { "Instructional Material Motivational } \\
\text { Survey» (IMMS) (Keller, 2010). }\end{array}$ \\
\hline $\begin{array}{c}\text { Evaluación objetos de RA } \\
\text { por los estudiantes }\end{array}$ & $\begin{array}{c}\text { Instrumento «ad hoc» con } \\
\text { construcción tipo Likert. }\end{array}$ \\
\hline
\end{tabular}

Tercera Fase: Conocer las posibilidades educativas que ofrece al alumno ser como productor de experiencias formativas apoyadas en RA.

En 1985 miembros del equipo de investigación realizaron la investigación "Evaluación de los usos del vídeo en la enseñanza» financiada por el Ministerio de Educación y Ciencia (López-Arenas y Cabero, 1990), con esta pretendíamos ver las posibilidades educativas que tendría diversas formas de utilizar el vídeo y una de ellas era su utilización como instrumento de conocimiento; es decir, la posibilidad que los alumnos se conviertan en productores de videomensajes y analicen de esta forma la realidad. Y ello es precisamente lo que perseguimos con esta fase de la investigación, que los alumnos se conviertan en productores de materiales educativos con estructura de RA. Con ello pretendemos alcanzar el objetivo 7 señalado anteriormente.

Su ejecución se llevó a cabo a través de diferentes etapas: selección de los grupos, formación de los grupos, trabajo en grupo por los alumnos para la elaboración de contenidos en formato RA y evaluación de la experiencia. Se llevó a cabo con alumnos de las universidades de Sevilla y Huelva que cursaban los estudios de Pedagogía y Maestros de Educación Infantil y Primaria.

FIGURA 3

Producción de objetos de RA por los estudiantes
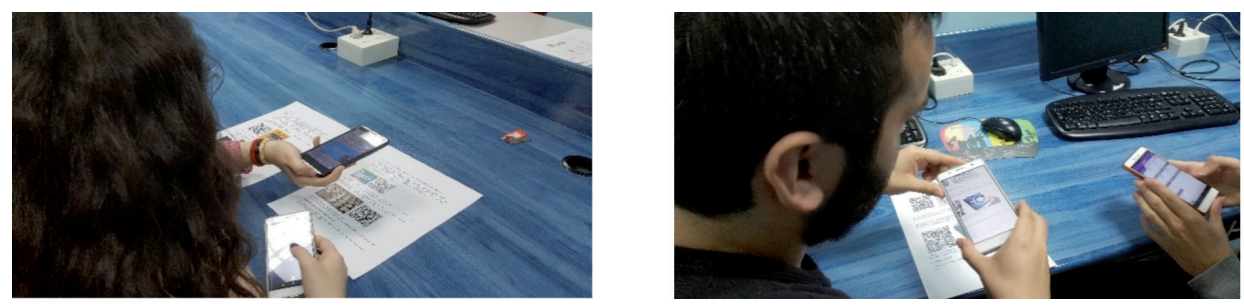
Cuarta Fase: Crear un entorno formativo bajo la arquitectura de la RA en formato libro electrónico, para la capacitación del profesorado universitario en el diseño, producción y utilización educativa de la RA.

Este material fue utilizado en cursos de formación del profesorado, que se vinieron desarrollando en diferentes universidades tanto nacionales como internacionales y puede ser descargado desde la siguiente url: http://grupotecnologiaeducativa.es/proyectorafodiun/index.php/difusion/curso-formacion-profesorado.

FIGURA 4

Material para la formación del profesorado en RA

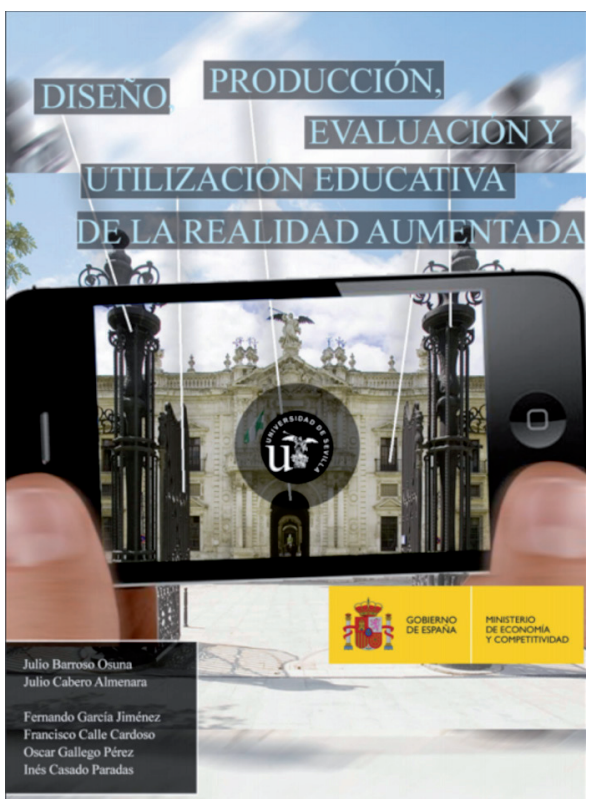

\subsection{Aportaciones desde el proyector RAFODIUM para el uso educativo de la RA}

Las diferentes investigaciones llevadas a cabo a través del proyecto RAFODIUM nos permiten apuntar algunas ideas para la utilización e incorporación de la RA a situaciones de enseñanza-aprendizaje.

- Los alumnos mostraron altos niveles de satisfacción al participar en experiencias de interacción con objetos de RA, así como también un alto grado de aceptación de las tecnologías de la RA. Al mismo tiempo, señalar que el modelo de aceptación de las tecnologías formulado por Davis (1989) se ha mostrado eficaz para conocer el grado de aceptación de las tecnologías de la RA por parte de los estudiantes, y como elemento para determinar la 
intención futura de utilización por ellos. Al mismo tiempo se encontraron relaciones significativas entre el nivel de satisfacción hacia la RA y el rendimiento académico obtenido por los estudiantes.

- La utilización de la RA es posible en diferentes disciplinas científicas, los resultados encontrados referidos a la motivación, grado de aceptación y rendimiento han sido idénticos en las distintas áreas de conocimiento donde se han llevado a cabo los estudios. En concreto, en las áreas de Ciencias de la Educación, Medicina, Bellas Artes, Arquitectura, Música e Ingeniería. Ello confirma lo apuntado en la primera parte del trabajo respecto a que ha sido utilizado en diferentes áreas de conocimiento con resultados significativos.

- Los estudios realizados ponen de manifiesto tres aspectos: a) la participación en experiencias de RA aumenta la motivación de los estudiantes medida a través del IMMs y de las diferentes dimensiones que lo conforman (confianza, atención, satisfacción y relevancia); b) se ha encontrado relación significativa entre el grado de motivación y el aumento del rendimiento, a mayor motivación (confianza. atención. satisfacción y relevancia) mayor rendimiento o recuerdo de la información alcanzada. De igual manera, se obtuvo una relación significativa y positiva entre la motivación y la evaluación efectuada de los objetos, y c) la motivación mostrada es superior cuando se convierten en productores.

- La participación en experiencias de producción de objetos en RA ha puesto de manifiesto que los estudiantes pueden convertirse en productores de estos recursos y que la realización de experiencias de este tipo aumenta la motivación del estudiante, el grado de aceptación de esta tecnología y el aprendizaje de los contenidos sobre los que versaron las experiencias.

- Tras la participación en las diferentes experiencias, los alumnos tienden a mostrar un alto nivel de intención de uso futuro de esta tecnología en su formación.

- El juicio de expertos permitió identificar los diez programas más usuales para la producción de objetos en RA: Arlab, Armedia, Arpa, ARTTolkit, Aurasma, Blippar, Designers artoolkit, Layar, Vuforia y Wikitude. De estos, los tres que fueron valorados como que requerían más conocimiento técnico para los usuarios potenciales fueron: Designers artoolkit, Vuforia y ARTTolkit; los tres con más complicación de navegación: Arlab, Designers artoolkit y Vuforia; los tres que permiten incorporar un mayor número de recursos: Vuforia, Vuforia y Layar; y los que son más fáciles de utilizar por los usuarios: Aurasma, Blippar y Arlab.

- Los alumnos valoraron muy positivamente los objetos producidos en RA, de ellos pueden obtenerse diferentes principios para el diseño de los materiales. Los requisitos que deben cumplir los objetos en RA para que sean usados desde un punto de vista educativo deben ser: 
- Los módulos de contenidos deben ser breves y directos, con duración de explotación seguida de no más de 10 minutos.

- Deben ser flexibles y simples, para poder adaptarse a las diferentes características y capacidades tecnológicas de los estudiantes.

- Tendencia a la organización simple desde el punto de vista tecnológico.

- Deben ser accesibles y tolerantes a los errores de manejo interactivo de los estudiantes, poseyendo un interfaz intuitivo que permita la corrección rápida de errores relacionados con la navegación y el uso del dispositivo.

- Tendencia a la producción multimedia, incorporando recursos de diferente tipología: audio, vídeo, imágenes 3D, animaciones, pdf, sitios web...

- Orientado hacia la acción, participación e interacción del usuario con el objeto producido.

- Comunicación y visibilidad: la portabilidad y la conectividad de estos dispositivos debería conducir a la creación de actividades colaborativas y permitir la posibilidad de compartir las contribuciones generadas en el proceso de aprendizaje.

- Contemplar en su producción que sean multiplataforma y, por tanto, adaptados a las características de los dispositivos de observación e interacción.

- La modalidad de libros y apuntes enriquecidos con objetos de RA ofrece bastantes posibilidades para ser incorporada a la formación universitaria. Los resultados encontrados han puesto de manifiesto que su utilización mejora el aprendizaje y despierta un verdadero grado de aceptación de la tecnología por parte de los estudiantes y los objetos producidos son valorados positivamente por los estudiantes. Nuestro trabajo también nos permite señalar que los apuntes enriquecidos mediante RA, son percibidos por los estudiantes como fáciles y flexibles de utilizar, mostrando al mismo tiempo una verdadera intención de utilizarlos en su formación.

\section{El proyecto de inNOVación en Ra en el Media lab del ilce (México)}

\subsection{El EDULAB ILCE}

Para prosperar en el siglo Xxi los estudiantes necesitan más que un aprendizaje académico tradicional, deben generar habilidades que les permitan prepararse para el futuro. En este sentido, hacia mediados del año 2014, el Instituto Latinoamericano de la Comunicación Educativa (ILCE), cuya sede está en Cd. México y cuenta con 14 países miembros, todos latinoamericanos, se replanteó los temas fundamentales hacia donde debería continuar trabajando y enfocando esfuerzos. Y se plasmó en el documento de Renovación del Instituto la creación del Laboratorio de Innovación Educativa. 
A partir de ese momento, con un grupo de trabajo realizó el primer planteamiento documental de un Laboratorio de Innovación Educativa nombrado EDULAB ILCE.

El propósito de su creación fue investigar y aplicar nuevas prácticas surgidas a partir de las redes digitales que responden a los grandes retos educativos de nuestro tiempo.

- Desarrollar experiencias para motivar las habilidades del siglo xxI, que permitan a los profesores adaptar sus competencias a los nuevos retos que se les presentan.

- Brindar apoyo a los gobiernos para generar alternativas de valor en los modelos 1:1 que permitan la usabilidad de los dispositivos, la transformación del espacio educativo y el rol docente/estudiante.

- Ayudar a los ciudadanos a disponer de entornos formativos que les den competencias para desarrollarse como parte activa y comprometida en la Sociedad del Conocimiento.

- Se contemplaba que los proyectos del laboratorio incidirían en alumnos, docentes y comunidades educativas, a favor de la educación en América Latina.

- Experimentar con tecnologías interactivas que promuevan formas colaborativas innovadoras de acceso y construcción del conocimiento y estilos narrativos.

- Profundizar en el "pensamiento de diseño" y facilitar el pasaje de una cultura del consumo reproductivo, a una de producción masiva de objetos, materiales y simbólicos.

- Investigar usos innovadores de las tecnologías para mejorar el aprendizaje de los jóvenes y los docentes en la cultura digital.

- Recuperar y potenciar aquellas prácticas innovadoras mediadas por tecnologías, que sean significativas para el campo de la educación y la formación en sentido amplio.

En esta fase inicial, las áreas de trabajo planteadas para el laboratorio fueron: Medios del Futuro, Tecnología Educativa, Diseño de Dispositivos, Cultura Digital.

Para enero de 2016, en el marco de su lanzamiento y generando espacios de conocimiento e intercambio, se ofreció la primera conferencia sobre la «cultura maker», y para el verano de 2016 por primera vez se ofreció un Summer Lab a más de 50 participantes.

El propósito era desarrollar conocimiento propio y específico sobre los avances de la tecnología para el aprendizaje que permita diseñar, aplicar, difundir, evaluar y proponer estrategias educativas innovadoras y pertinentes a la multidiversidad de los contextos latinoamericanos.

Los objetivos que persigue el citado proyecto son: 
1. Realizar aplicaciones piloto con diversas herramientas, programas y/o software para observar resultados e impactos de su utilización en estrategias educativas.

2. Promover en las Instituciones Normalistas (de preparación de maestros) la implementación del modelo que permita a los futuros docentes adquirir ideas, metodologías y conocimientos sobre las nuevas pedagogías.

3. Promover las competencias del siglo XXI a fin de que los maestros adapten su práctica docente a los retos y características que sus alumnos enfrentarán en el futuro.

4. Formar a los maestros en la planeación, desarrollo y evaluación de estrategias didácticas con el uso de diversos medios, herramientas y dispositivos tecnológicos.

5. Conformar comunidades de docentes-investigadores-creadores de estrategias educativas innovadoras.

6. Recuperar y potenciar aquellas prácticas innovadoras mediadas por tecnologías que sean significativas en los contextos socioeducativos.

7. Conformar redes de colaboración con instituciones y empresas que compartan el interés por la mejora de la educación.

8. Experimentar con tecnologías interactivas que promuevan formas colaborativas innovadoras de acceso y construcción del conocimiento y estilos narrativos.

9. Profundizar en el "pensamiento del diseño" base del pensamiento innovador y facilitar el tránsito de una cultura tradicional del consumo reproductivo a una de producción de conocimiento.

A través de metodologías activas e inclusivas, como el aprendizaje basado en retos, el aprendizaje basado en proyectos, el aprendizaje basado en problemas, parafraseando a John Dewey a principios del siglo xx, les damos a los alumnos y, en este caso, también a los maestros, cosas que hacer, para aprender. Implementamos a través de las nuevas pedagogías como "maker", "do it your self (DYS)", actividades que permitan potenciar el pensamiento "STEAM» (ciencia, tecnología, Ingeniería, artes y matemáticas), ya que como diría Edgar Morin (2001) «aprender hoy es dialogar con la incertidumbre».

EDULAB ILCE cuenta con más de 40 talleres de dos horas cada uno, que integran aprendizajes del siglo XXI a lo largo de un ciclo escolar, se generan productos de robótica, programación, storytelling, emprendimiento, cultura digital, ciencias y artes, principalmente. Con los cuales se puede cubrir todo un ciclo escolar completo, que en el caso de México abarca 40 semanas de clases.

\subsection{RA EDULAB}

Somos seres tridimensionales, nuestro cerebro es la computadora 3D más poderosa, hemos evolucionado y vivimos en un mundo de tercera dimensión. Los 
seis siglos de tradición gutemberiana nos habituaron a la información plana de una hoja de papel, que ahora se ha trasladado hacia la pantalla del ordenador. La realidad aumentada es la mejora de la realidad "vista» por el usuario a través de un dispositivo que presenta información adicional. Los dispositivos deben tener una "forma de visualización» generalmente a través de una cámara, ya sea de una computadora portátil, una tableta o un teléfono inteligente. Cuando se superpone información a las imágenes reales en forma de gráficos, sonidos, animaciones, vídeo, coordenadas GPS y comentarios, estamos ampliando con la RA la representación visual del mundo que nos rodea. De esta forma, el mundo real se vuelve interactivo y cede al procesamiento digital.

Como ya se indicó la RA no debe confundirse con la realidad virtual, ya que los límites de cada concepto están claramente demarcados. Mientras que la realidad virtual denota una realidad que existe solo en el mundo virtual (en línea), la realidad aumentada, por otro lado, mantiene el mundo real, pero solo le agrega una capa digital o lo amplifica. En otras palabras, la RA le brinda información real sin cambiar o desplazar el mundo real que está experimentando.

La integración de la tecnología de RA en el aprendizaje está adquiriendo fuerte presencia. Por ello desde el Centro de Investigación y de Estudios Avanzados del Instituto Politécnico Nacional (Cinvestav), a través su Agencia de Comercialización de Conocimiento de esa institución, lanzaron el Concurso IDi4Biz® emprendedores dirigido a sus estudiantes y egresados de posgrado para la generación de propuestas de proyectos de emprendimiento, con el objeto de identificar, madurar y estructurar ideas emprendedoras, de sus estudiantes y egresados de posgrado, para la puesta en marcha de nuevas empresas de base tecnológica fundamentadas en el conocimiento y tecnologías desarrolladas.

En este contexto, en 2013 uno de los proyectos ganadores, merecedor de un premio económico, de la asesoría para la maduración y del establecimiento de su plan de negocios, fue KuruchuSoft, aplicación móvil de Realidad Aumentada que nace con la idea de convertir el aprendizaje de libros en una experiencia interactiva a los libros de texto gratuito que la Secretaría de Educación Pública de México distribuye a lo largo y ancho del país (Figura 5). Libros o apuntes enriquecidos con objetos de RA que de acuerdo con diferentes estudios mejoran el aprendizaje, la motivación y que es una tecnología con una fuerte aceptación por los estudiantes (Nadolny, 2016; Cheng, 2017; Hung, Chen y Huang, 2017; Garay, Tejada y Maíz, 2017; Barroso, 2018). 
FIGURA 5

Imágenes del programa
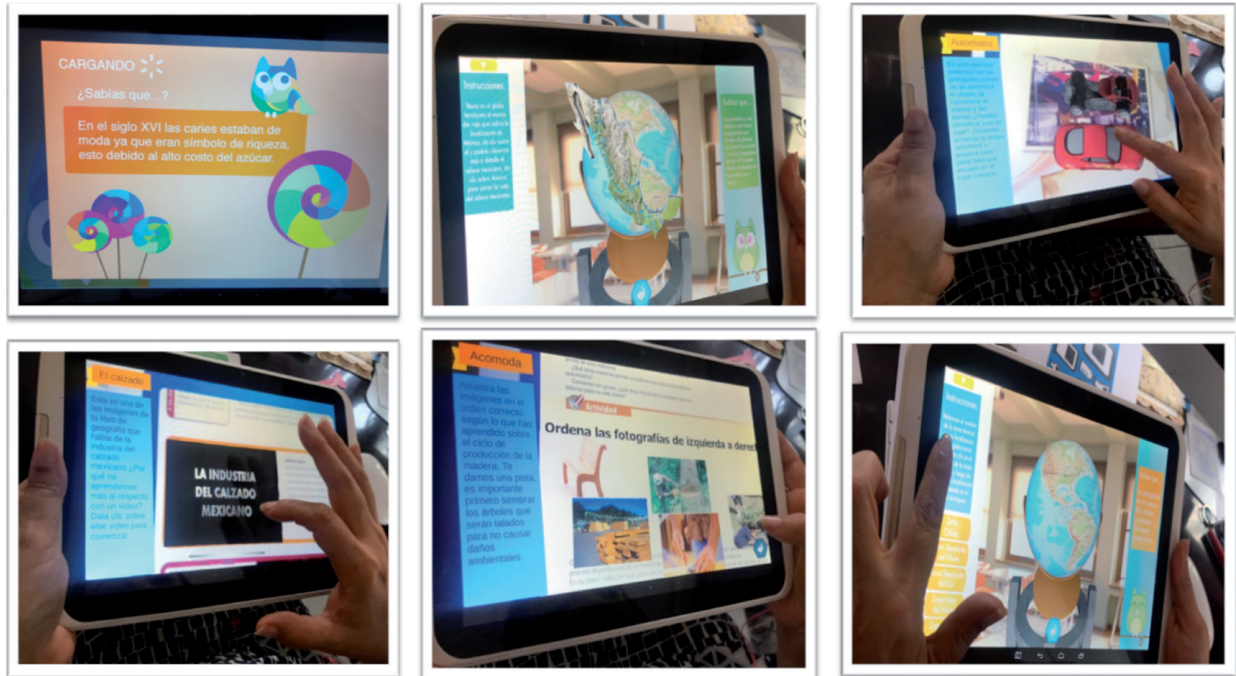

En la Figura 6, se presenta un código QR, con el cual se puede acceder a una demostración del funcionamiento y posibilidades del programa.

FIGURA 6

Código QR de explicación del funcionamiento del programa

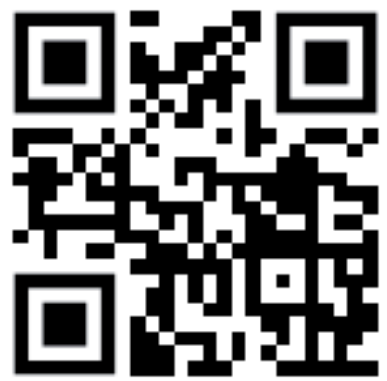

Con el fin de incrementar el aprendizaje y conocimientos de los millones de niños inscritos en primaria, de lograrse su implementación a nivel federal, se elaboró una propuesta de libros enriquecido con objetos de RA. 
La aplicación se instala y no requiere conexión de Internet, basta con descargar la app en la tableta y utilizar la cámara para enfocar imágenes del propio libro, para que aparezcan videos, animaciones, textos. Se inició con el libro de quinto grado de Geografía, una de las áreas con más bajo resultado estudiantil de acuerdo con la prueba ENLACE 2011, aunque la intención a mediano plazo fue continuar con el resto de asignaturas del nivel como Ciencias Naturales, Matemáticas e Historia y así continuar con los libros de otros grados.

Se modificaron y enriquecieron 57 de las 100 imágenes que contiene el libro, como volcanes de México, que lucen como montañas dibujadas, pero gracias a la aplicación adquieren relieve, se observan como una figura real, tomada por satélite, además se muestra su descripción, localización y altura.

Hay actividades en el libro que por lo general los alumnos no le dan la continuidad para realizarla, como es el caso de que deben observar durante seis semanas los cambios que sufre la sombra de un árbol. Por lo que la RA ayuda, generando un árbol en 3D y con un clic cambia la sombra en una especie de línea de tiempo.

Este prototipo fue probado con más de 200 alumnos de diversas primarias del Distrito Federal y el Estado de México, con la idea de conocer el interés que les despierta y si se genera un impacto en el aprendizaje. El estudio piloto se llevó a cabo analizando diferentes variables, que iban desde la facilidad de desplazamiento y navegación por el objeto, su facilidad de manejo y el grado de aceptación que el objeto producía en los estudiantes. Y debemos señalar que los niveles de satisfacción mostradon por los estudiantes fueron verdaderamente significativos, y que de manera general presentaremos posteriormente. De todas formas, esta es una de las limitaciones que consideramos del proyecto: la necesidad de establecer una evaluación más formalizada.

Un clip de vídeo del funcionamiento del programa puede observarlo en la siguiente dirección web, puede ver un vídeo del funcionamiento del programa:

En esta alianza, el EDULAB ILCE fue responsable de plantear una propuesta para cada aprendizaje esperado, considerando el programa de estudio de la asignatura, el enfoque, los recursos didácticos, la secuencia didáctica, el análisis de la práctica docente, información adicional para saber más y la retroalimentación, integrando de acuerdo a cada objeto de realidad aumentada, considerando saberes previos, nuevos retos, práctica y evaluación. Realizando un guion tecnopedagógico de secuencia didáctica para cada uno de los temas.

Como el ILCE había participado en la incorporación de contenidos y capacitación docente del Programa de Inclusión Digital de la SEP (Secretaría de Educación Pública del Gobierno de México) donde se entregaron tabletas a los estudiantes de quinto grado de primaria, mismas que funcionaban con sistema Android, por lo que el algoritmo de esta app de RA se desarrolló con las especificaciones para su óptimo funcionamiento en ella.

La prueba piloto realizada nos permitió identificar una serie de ventajas y de desafíos para la incorporación en la enseñanza (Figura 7). Por lo que se refiere a las ventajas que nos encontramos tras su introducción, las podemos encuadrar en las siguientes: 
- Los estudiantes cuentan con las habilidades para interactuar con la aplicación.

- Se alcanzaron los aprendizajes esperados en el tema.

- Generó interés en el estudiante, al referirse a experiencias reales, contextualizada, multimedia, interactiva y tridimensionales.

- Promueve la interacción educativa, la colaboración, movimiento y apoyo entre los estudiantes.

\section{FIGURA 7}

Interacción de los estudiantes con el programa
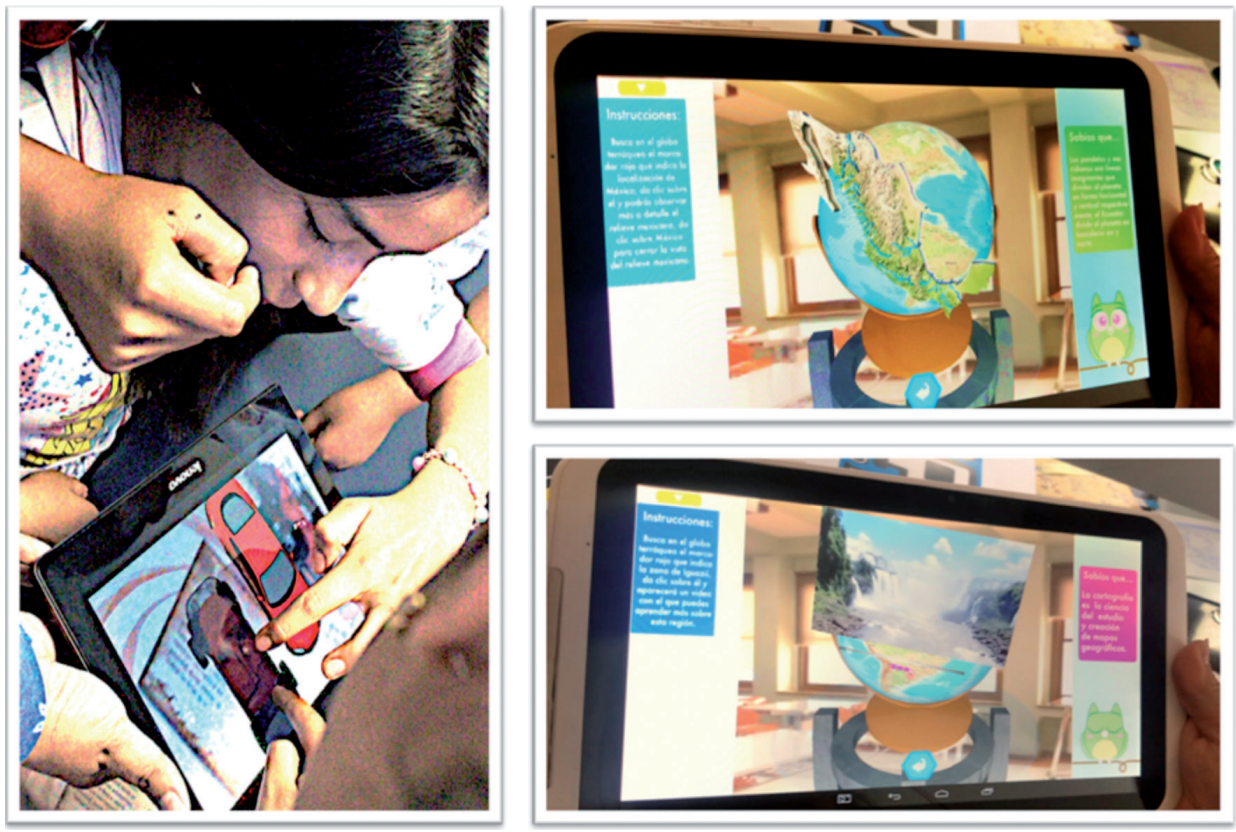

De todas formas, también creemos que nos encontramos con una serie de desafíos sobre los que debemos reflexionar antes de su incorporación a la práctica educativa, y que concretamos en las siguientes:

- La innovación y el atractivo de la tecnología pueden dar como resultado una menor atención dedicada a la calidad y el contenido, que es de gran importancia para los objetivos educativos.

- No ha sido probado que sea especialmente más beneficioso el aprendizaje esperado con la utilización de la RA. 


\section{A MANERA DE CONCLUSIÓN}

Las dos experiencias realizadas nos permiten obtener una serie de conclusiones, que especificamos en los siguientes términos:

- Es una tecnología emergente que está penetrando fuertemente en nuestro sistema educativo, teniendo ya aplicaciones que pueden ser utilizadas en los diferentes niveles de enseñanza.

- Es una tecnología que puede ser utilizada en diferentes áreas y disciplinas del conocimiento.

- La modalidad de libros y apuntes enriquecidos con objetos de RA es una modalidad que perfectamente puede ser incorporada a la enseñanza. Modalidad que nos lleva a sugerir, de acuerdo con otros autores (Alkhattabi, 2017; Joo, Martínez, García y García, 2017), que perfectamente podrán ser utilizados en acciones de e-learning, para favorecer la realización de acciones prácticas por los estudiantes y facilitar el aprendizaje en contextos reales en los entornos virtuales de formación.

- Se hace necesario que se creen centros de producción de estos objetos tecnológicos, pues su calidad es determinante para que los estudiantes muestren grados de aceptación de esta tecnología.

- Su producción implica no solo el diseño tecnológico del objeto, sino fundamentalmente un diseño pedagógico.

- Su incorporación a la enseñanza pasa por la formación del profesorado para su utilización.

- Y se hace necesario incrementar su investigación, para buscar pistas sobre su diseño y utilización, y para conformar un marco teórico que justifique sus diferentes formas de utilización en la enseñanza.

\section{REFERENCIAS BIBLIOGRÁFICAS}

Aguayo, C.; Cochrane, Th. y Narayan, V. (2017). Key themes in mobile learning: Prospects for learner-generated learning through AR and VR. Australasian Journal of Educational Technology, 33 (6), 27-40.

Akcayr, M. y Akcayr, G. (2017). Advantages and challenges associated with augmented reality for education: A systematic review of the literatura. Educational Research Review, 20, 1-11.

Alkhattabi, M. (2017). Augmented Reality as E-learning Tool in Primary Schools' Education: Barriers to Teachers' Adoption. International Journal of Emerging Technologies in Learning, 12 (2), 91-100.

Barba, R.; Yasaca, S. y Manosalvas, C. (2015). Impacto de la realidad aumentada móvil en el proceso enseñanza-aprendizaje de estudiantes universitarios del área de medicina. Investigar con y para la Sociedad, vol 3. Cádiz: Bubok Publishing S.L; 1421-1429.

Barroso, J. (2018). The technological scenarios in Augmented Reality (AR): educational possibilities in university studies. Aula Abierta, 47 (3), 327-333. 
Barroso, J. y Cabero, J. (2016). Evaluación de objetos de aprendizaje en realidad aumentada: estudio piloto en el grado de Medicina. Enseñanza \& Teaching, 34 (2), 149-167.

Barroso, J. y Gallego, O. (2017). Producción de recursos de aprendizaje apoyados en Realidad Aumentada por parte de los estudiantes de magisterio. Edmetic. Revista de Educación Mediática y TIC, 6, 1, 23-38.

Bressler, D. M. y Bodzin, A. M. (2013). A mixed methods assessment of students' flow experiences during a mobile augmented reality science game. Journal of Computer Assisted Learning, 29 (6), 505-517.

Brigham, T. (2017). Reality Check: Basics of Augmented, Virtual, and Mixed Reality. Medical reference services quarterly, 36 (2), 171-178. http://dx.doi.org/10.1080/02763869.2017 .1293987.

Buitrago-Pulido, R. D. (2015). Incidencia de la realidad aumentada sobre el estilo cognitivo: caso para el estudio de las matemáticas. Educación y Educadores, 18 (1), 27-41.

Cabero, J.; Fernández, B. y Marín, V. (2017). Dispositivos móviles y realidad aumentada en el aprendizaje del alumnado universitario. RIED. Revista Iberoamericana de Educación a Distancia, 20 (2), 167-185. doi: http://dx.doi.org/10.5944/ried.20.2.17245.

Cabero, J. y otros (2016). Realidad Aumentada y educación. Barcelona: Octaedro.

Cabero, J. y García, F. (Coords.) (2016). Realidad aumentada. Tecnología para la formación. Madrid: Síntesis.

Cabero, J. y otros (Coords.) (2018). La realidad aumentada como herramienta educativa. Aplicación a la educación infantil, primaria, secundaria y bachillerato. Madrid: Paraninfo.

Carozza, L.; Tingdahi, D.; Bosché, F. y Gool, L. (2014). Markerless Vision-Based Augmented Reality for Urban Planning. Computer-Aided Civil and Infrastructure Engineering, 00, $1-16$.

Chang, S. y Hwang, G. (2018). Impacts of an augmented reality-based flipped learning guiding approach on students' scientific project performance and perceptions. Computers E Education, 125, 226-239. https://doi.org/10.1016/j.compedu.2018.06.007.

Chen, Ch. y Wang, Ch. (2015). Employing Augmented-Reality-Embedded Instruction to Disperse the Imparities of Individual Differences in Earth Science Learning. J Sci Educ Technol, 24, 835-847.

Cheng, K.-H. (2017). Reading an augmented reality book: An exploration of learners' cognitive load, motivation, and attitudes. Australasian Journal of Educational Technology, 33 (4), 53-69. https://doi.org/10.14742/ajet.2820.

Chursches, A. (2008). Taxonomía de Bloom para la era digital. Eduteka. Recuperado de http://www.eduteka.org/TaxonomiaBloomDigital.php-.

Davis, F. (1989). Perceived usefulness, perceived ease of use, and user acceptance of information technology. MIS Quarterly, 13 (3), 319-340. doi: 10.2307/249008.

De la Torre Cantero, J. y otros (2013). Entorno de aprendizaje ubicuo con realidad aumentada y tabletas para estimular la comprensión del espacio tridimensional. RED, Revista de Educación a Distancia, 37. Recuperado de http://www.um.es/ead/red/37.

Di Serio, A. y otros (2013). Impact of an augmented reality system on students' motivation for a visual art course. Computers \& Education, 68, 586-596.

Fernández, B. (2018). La utilización de objetos de aprendizaje de realidad aumentada en la enseñanza universitaria de educación primaria. International Journal of Educational Research and innovation (IJERI), 9, 90-104. 
Fonseca, D.; Redondo, E. y Valls, F. (2016). Motivación y mejora académica utilizando realidad aumentada para el estudio de modelos tridimensionales arquitectónicos. Education in the Knowledge Society, EKS, 17 (1), 45-64.

Gallego, O.; Barroso, J. y Marín, V. (2018). Análisis de la motivación de los estudiantes universitarios como productores de recursos educativos utilizando la Realidad Aumentada. Espacios, 39 (25), 8 páginas.

Garay, U.; Tejada, E. y Maiz, I. (2017). Valuation of the educational objects with augmented reality: experience with posgraduates students. Pixel-Bit. Revista de Medios y Educación, 50, 19-31. doi: http://dx.doi.org/10.12795/pixelbit.2017.i50.01.

Han, J.; Jo, M.; Hyun, E. y So, H. (2015). Examining young children's perception toward augmented reality-infused dramatic play. Education Technology Research Development, 63, 455-474.

Hung, Y.; Chen, C. y Huang, S. (2017). Applying augmented reality to enhance learning: a study of different teaching materials. Journal of Computer Assisted, 33, 252-266. doi: 10.1111/jcal.12173.

Ibáñez, M. y Delgado, C. (2018). Augmented reality for STEM learning: A systematic review. Computers E Education, 123, 109-123. https://doi.org/10.1016/j.compedu.2018.05.002.

Jamali, S. y otros (2015). Utilising mobile-augmented reality for learning human anatomy. Procedia-Social and Behavioral Sciences, 197, 659-668.

Johnson, L.; Adams Becker, S.; Cummins, M.; Estrada, V.; Freeman, A. y Hall, C. (2016). NMC Horizon Report: 2016 Higher Education Edition. Austin, Texas: The New Media Consortium.

Joo, J.; Martínez, F.; García, J. y García, F. (2017). Augmented reality and pedestrian navigation through its implementation in m-learning and e-learning: Evaluation of an educational program in Chile. Computers \& Education, 111, 1-17. http://dx.doi.org/10.1016/j. compedu.2017.04.0030360-1315/.

Keller, J. M. (2010). Motivational design for learning and performance. New York: Springer Science+Business.

Lin, T.; Been-Lirn, H.; Li, N.; Wang, H. y Tsa, Ch. (2013). An investigation of learners' collaborative knowledge construction performances and behavior patterns in an augmented reality simulation system. Computers \& Education, 68, 314-321.

Marín, V. (2017). The augmented reality in the educational sphere of student of degree in chilhood education. Case study. Píxel-Bit. Revista de Medios y Educación, 51, 7-19. doi: http://dx.doi.org/10.12795/pixelbit.2017.i51.01.

Martínez, S. y Fernández, B. (2018a). Objects of augmented reality: perceptions of Pedagogy students. Píxel-Bit. Revista de Medios y Educación, 53, 207-220. doi: http://dx.doi. org/10.12795/pixelbit.2018.i53.14.

Martínez, S. y Fernández, B. (2018b). Objetos de Realidad Aumentada: percepciones del alumnado de Pedagogía. Pixel-Bit. Revista de Medios y Educación, 53, 207-220.

Nadolny, L. (2016). Interactive print: The design of cognitive tasks in blended augmented reality and print documents. British Journal of Educational Technology, 00 (00), 1-10. doi: 10.1111/bjet.12462.

Pejoska-Laajola, J.; Reponen, S.; Virnes, M. y Leinonen, T. (2017). Mobile augmented communication for remote collaboration in a physical work context Jana. Australasian Journal of Educational Technology, 33 (6), 11-25. 
Piqueras, E. y González-Calero, J. A. (2018). Incidencia de la realidad aumentada en la enseñanza de la historia. Una experiencia en tercer curso de educación primaria. Enseñanza \& Teaching, 36 (1), 23-39. doi: https://doi.org/10.14201/et20183612339.

Prendes, C. (2015). Realidad aumentada y educación: análisis de experiencias prácticas. Pixel-Bit. Revista de Medios y Educación, 46, 187-203.

Santos, M.; Wolde, A.; Taketomi, T.; Yamamoto, G.; Rodrigo, M.; Sandor, Ch. y Kato, H. (2016). Augmented reality as multimedia: the case for situated vocabulary learning. Research and Practice in Techology Enhanced Learning, 11 (4), 1-23.

Sevillano, M. L. y Vázquez-Cano, E. (2015). Modelos de investigación en contextos ubicuos y móviles en Educación superior. Madrid: McGraw-Hill-UNED.

Tecnológico de Monterrey (2015). Reporte EduTrends. Radar de Innovación Educativa 2015. Monterrey: Tecnológico de Monterrey.

Tecnológico de Monterrey (2017). Reporte EduTrends. Realidad Aumentada y virtual. Monterrey: Tecnológico de Monterrey.

Tekederea, H. y Gökera, H. (2016). International Journal of Environmental \& Science Education, 11 (16), 9469-9481.

Vázquez-Cano, E. y Sevillano, M. L. (Eds.) (2015). Dispositivos digitales móviles en educación. Madrid: Narcea.

Villalustre, L. y Del Moral, M. E. (Coords.) (2016). Experiencias interactivas con realidad aumentada en el aula. Barcelona: Octaedro.

Wu, H.-S.; Wen-Yu, S.; Chang, H.-Y. y Liang, J. (2013). Current status, opportunities and challenges of augmented reality in education. Computers E Education, 62, 41-49. 